\title{
ARID1A mutations and expression loss in non-small cell lung carcinomas: clinicopathologic and molecular analysis
}

\author{
Yin P. Hung $\mathbb{1}^{1,2} \cdot$ Amanda Redig $^{3} \cdot$ Jason L. Hornick $\mathbb{B}^{1} \cdot$ Lynette M. Sholl $\mathbb{D}^{1}$ \\ Received: 24 April 2020 / Revised: 30 May 2020 / Accepted: 1 June 2020 / Published online: 22 June 2020 \\ (c) The Author(s), under exclusive licence to United States \& Canadian Academy of Pathology 2020
}

\begin{abstract}
A subset of non-small cell lung carcinomas harbors mutations in ARID1A, a component of the SWI/SNF complex implicated in modulating response to immunotherapy in diverse tumors. This study characterized the spectrum of ARIDIA mutations and expression by targeted sequencing and immunohistochemistry, respectively. In a consecutive series of 2440 non-small cell lung carcinomas, ARIDIA mutations were present in $184(7.5 \%)$, within which $69 \%$ harbored loss-of-function mutations. Of 139 ARID1A-mutated tumors available for immunohistochemistry, ARID1A expression was aberrant in 64 (46\%), including diffuse complete loss in 13 (9\%), diffuse diminished expression in 17 (12\%), and heterogeneous loss with a geographic or interspersed pattern in 34 (25\%). Complete loss of ARID1A expression correlated with ARIDIA prematuretruncating mutations with evidence of biallelic inactivation. Both ARIDIA mutations and aberrant expression correlated with a lack of EGFR mutations, frequent TP53 mutations, and increased mutational burden. ARIDIA-mutant tumors showed similar overall survival compared with ARIDIA-wild-type tumors; however, among patients with ARIDIA-mutant tumors, aberrant ARID1A expression correlated with worse overall survival. Lung tumors with diffuse loss of ARID1A expression were predominantly adenocarcinomas, poorly differentiated, almost exclusively from smokers, and enriched for mismatch repair deficiency. Geographic heterogeneous ARID1A loss was notable in three tumors, including an adenocarcinoma showing fetal-like differentiation in areas with ARID1A loss. Overall, loss of ARID1A expression at the protein level is seen in fewer than $2 \%$ of non-small cell lung carcinomas but is associated with distinct clinicopathologic features. Our findings suggest a need for caution in interpretation of the functional significance of ARIDIA mutations from sequencing data.
\end{abstract}

Presented in part at the 107th Annual Meeting of the United States and Canadian Academy of Pathology (USCAP), Vancouver, BC;

March 2018.

Supplementary information The online version of this article (https:// doi.org/10.1038/s41379-020-0592-2) contains supplementary material, which is available to authorized users.

Lynette M. Sholl

lmsholl@bwh.harvard.edu

1 Department of Pathology, Brigham and Women's Hospital and Harvard Medical School, Boston, MA, USA

2 Department of Pathology, Massachusetts General Hospital and Harvard Medical School, Boston, MA, USA

3 Department of Medical Oncology, Dana-Farber Cancer Institute and Harvard Medical School, Boston, MA, USA

\section{Introduction}

AT-rich interacting domain containing protein 1A (ARID1A; also known as BAF250a and SMARCF1) is a key noncatalytic component in the SWItch/sucrose non-fermentable (SWI/SNF) chromatin remodeling complex [1]. Of all human tumors, $20 \%$ harbor mutations involving members of the SWI/SNF complex, including ARIDIA, ARID1B, ARID2, PBRM1, SMARCA4, and others [2]. Mutations in ARIDIA are frequent across cancer types, present in over $30-50 \%$ of ovarian and endometrial carcinomas [3-5], 10\% of hepatocellular [6, 7] and bladder carcinomas [8], and 5-10\% of colorectal [8], gastric [9, 10], and non-small cell lung carcinomas $[8,11,12]$. The functional significance of ARID1A is context-dependent. ARID1A functions primarily as a tumor suppressor in most tumor types, including ovarian clear cell and endometrioid carcinomas [3, 4]. ARID1A loss is associated with PI3K-Akt pathway activation in ovarian clear cell carcinomas [13], resistance to trastuzumab in HER2-positive breast carcinomas [14], and impairment in 
enhancer-mediated gene regulation in murine colorectal tumor models [15]. In contrast, in hepatocellular carcinoma, ARID1A acts as an oncogene in tumor initiation, but as a tumor suppressor in subsequent maintenance and metastasis [16].

Data on the functional and clinical significance of ARIDIA mutations in non-small cell lung carcinomas are limited. Studies suggest that disruption of different members of the SWI/SNF complex may have similar implications for lung cancer phenotype [17]. In particular, mutations in SMARCA4 are present in $6-11 \%$ of non-small cell lung carcinomas [12, 18, 19]; SMARCA4 mutations and expression loss have been implicated in the pathogenesis of a subset of aggressive thoracic sarcomas [20-22] and lung adenocarcinomas [18, 19, 23-27]. Given the nuanced context-dependent functions of ARID1A, it is nonetheless difficult to infer the impact of ARIDIA alterations based on studies from other tumor types or SWI/SNF members. Notably, ARIDIA may be included as part of multigene panels for targeted next-generation sequencing [28]. A recent study on plasma DNA from 185 patients with treatment-naive lung adenocarcinoma has identified ARIDIA mutations in $12 \%$ of patients, with co-occurrence of other oncogenic mutations such as KRAS and EGFR [29]. Given the preclinical data on targeting ARIDIA-mutant tumors with EZH2 inhibitors [30] and emerging data on implications of immunotherapy outcomes [31-33], we anticipate a growing need to detect, interpret, and understand the impact of $A R I D I A$ alterations in diverse tumors including non-small cell lung carcinomas. This study aims to characterize the spectrum of ARIDIA genetic alterations, correlate ARIDIA mutations with expression by immunohistochemistry, and assess the clinicopathologic significance of ARIDIA mutations and expression loss in nonsmall cell lung carcinomas.

\section{Materials and methods}

\section{Case selection and clinicopathologic evaluation}

The study was approved by the Institutional Review Boards at Brigham and Women's Hospital and Dana-Farber Cancer Institute and included a consecutive series of 2440 patients with their non-small cell lung carcinomas analyzed by targeted next-generation sequencing assays at the Center for Advanced Molecular Diagnostics, Brigham and Women's Hospital, between January 2014 and December 2017. This study included patients diagnosed with non-small cell lung carcinomas (adenocarcinoma, squamous cell carcinoma, adenosquamous carcinoma, and pleomorphic/giant cell/ spindle cell carcinoma); while patients with other diagnoses including small cell carcinoma, other neuroendocrine tumors (carcinoid tumors and large cell neuroendocrine carcinoma), combined small cell or large cell neuroendocrine with non-small cell carcinomas, and adenoid cystic carcinoma were excluded. Clinical features recorded in the DFCI/BWH Oncology Data Retrieval Systems (OncDRS) for the entire cohort included sex, age at molecular diagnosis, and survival/last follow-up. For the subset of patients with ARIDIA-mutant tumors, age at pathologic diagnosis, smoking history, and clinical stage (based on the American Joint Committee on Cancer Staging Manual 7th edition) were recorded.

\section{Targeted next-generation sequencing and variant interpretation}

Targeted next-generation sequencing (OncoPanel) was performed as previously described [34, 35], using DNA (at least $50 \mathrm{ng}$ ) extracted from formalin-fixed paraffin-embedded whole-tissue sections, solution-based hybrid capture with Illumina HiSeq 2500 (San Diego, CA), and a custom set of Agilent SureSelect capture probes (Santa Clara, CA). Over the 4-year study period, three versions of the in-house sequencing panel were used, targeting 275 genes covering $757,787 \mathrm{bp}$ in $1305(50.6 \%)$ cases, 298 genes covering 831,033 bp in $739(28.7 \%)$ cases, and 447 genes covering $1,315,708 \mathrm{bp}$ in $535(20.7 \%$ ) cases (a total of 2579 cases from 2440 patients). Sample reads (with mean target coverage of at least 50x) were analyzed using a custom bioinformatics pipeline with Picard and BWA (Broad Institute, Cambridge, MA) for alignment, VisCap Cancer (Dana-Farber Cancer Institute) for copy number variants, BreaKmer for structural variants, MuTect and GATK indelocator (Broad Institute) for single-nucleotide and insertion-deletion variants, and Oncotator and Integrated Genome Viewer (Broad Institute) for annotation.

For interpretation of ARIDIA variants, we excluded synonymous variants, single-nucleotide variants with a minor variant allele frequency of $>0.1 \%$ in the Exome Sequencing Project database (University of Washington, Seattle, WA), variants listed in the genome Aggregation Database (gnomAD; Broad Institute, Cambridge, MA), and suspected private germline variants based on the allele frequencies. Variants listed in the Catalogue of Somatic Mutations in Cancer (COSMIC) database (Wellcome Trust Sanger Institute, UK) were rescued. ARIDIA variants were interpreted based on tumor percentage and associated copy number alterations if present. Nonsense, frameshift, conserved splice site, and structural rearrangement/truncating variants were classified as loss-of-function (LOF) mutations. In tumors with known driver oncogene (such as KRAS or $E G F R$ ) mutations, an ARIDIA mutation clonality index was derived by normalizing the variant allele frequency of ARIDIA relative to that of the driver mutation. Samples 
with $<20 \%$ tumor were excluded from this analysis. We defined an ARIDIA mutation with a clonality index of $\geq 0.6$ as truncal and $<0.6$ as sub-clonal. Given the relative lack of robust informatics tools for assessing clonal architecture in solid tumors undergoing targeted next-generation sequencing, we determined that this cutoff point was a reasonable discriminator between truncal and sub-clonal variants, based on empiric data derived from whole-genome sequencing studies in myeloid tumors [36]. Clonality was indeterminate in tumors lacking driver mutations. Tumor mutational burden (TMB) was derived by tabulating the total number of non-synonymous missense and small insertion-deletion variants relative to the megabases $(\mathrm{Mb})$ sequenced. Copy number loss was interpreted by initial pathology review based on copy number VisCap plots in $\log _{2}$ ratio values relative to the genome baseline. Genomic instability index was calculated by tabulating the total number of copy number variants relative to the $\mathrm{Mb}$ sequenced. Copy-neutral loss of heterozygosity of ARIDIA was defined by showing either a presumed somatic variant allele frequency of $\geq 0.7$ or a clonality index of $\geq 1.4$, in the absence of a detectable copy change. Biallelic inactivation was defined as the presence of two LOF mutations (with no evidence of being in cis), one LOF mutation plus one copy deletion by copy number analysis, or one LOF mutation plus suspected copy-neutral loss of heterozygosity based on allele fraction of known polymorphisms. Cases were considered insufficient for this analysis when tumor content was estimated at $<20 \%$ (the validated lower limit for copy number detection) or when sample quality (reflected in low mean target coverage or high sequencing noise) precluded confident assessment of copy number alterations or quantification of variant alleles. Assessment of mismatch repair (MMR) deficiency was performed on sequencing data using an in-house clinically validated algorithm based on the number of insertion-deletion mutations involving homopolymer repeats [37].

\section{Immunohistochemistry}

Immunohistochemistry for ARID1A was performed on 4- $\mu$ mthick formalin-fixed paraffin-embedded whole-tissue sections using a rabbit antihuman ARID1A polyclonal antibody (1:500 dilution; HPA005456, Sigma) following pressure cooker antigen retrieval $(0.01 \mathrm{M}$ citrate buffer $\mathrm{pH}$ 6.0). Immunohistochemistry for ARID1A was performed on all ARIDIA-mutant non-small cell lung carcinomas with available material (143 cases from 139 patients), along with 40 ARIDIA-wild-type non-small cell lung carcinomas. ARID1A immunohistochemistry was reviewed by two pathologists (YPH and LMS), and a consensus score was recorded; both were blind to the ARIDIA molecular data at the time of the immunohistochemistry review. The percentage of cells with altered ARID1A expression and the pattern of loss were noted. Complete ARID1A loss was defined as the absence of perceptible ARID1A nuclear staining, while diminished ARID1A expression was defined as barely perceptible to moderate decrease in ARID1A nuclear staining, and ARID1A immunoreactivity was considered intact when there was no perceptible change in ARID1A nuclear staining in the tumor cells as compared with background control (stromal or inflammatory) cells. Diffuse loss of expression was defined as aberrant staining in at least $90 \%$ of cells; whereas heterogeneous loss of expression was defined as aberrant staining in $<90 \%$ of cells.

\section{Statistical analysis}

The landscape of ARIDIA alterations and mutation maps were illustrated using Microsoft Excel 2016 and Illustrator for Biological Sequences version 1.0.3 [38]. Statistical analysis was performed using GraphPad InStat version 3.1 and GraphPad Prism version 5.02 (LaJolla, CA). Categorical and non-categorical data were analyzed using chi-square tests and Mann-Whitney $U$ tests, respectively. For patients with ARIDIA-mutant tumors and the remainder of the cohort, survival was defined as the time of pathologic diagnosis and the time of molecular diagnosis, respectively, to the time of death from any cause or to the time of last clinical follow-up, at which point the data were censored. For assessment of mutational hotspots, a $P$ value was calculated at each amino acid position in comparison with the expected value based on the Poisson distribution $\left(\mu^{x} e^{-\mu} / x !\right)$. Statistical significance was defined by a $P$ value $<0.05$, with Bonferroni correction used in multiple comparisons.

\section{Results}

\section{Clinicopathologic and molecular features of our study cohort}

Our institutional cohort comprised 2440 patients (Supplementary Fig. 1), including 1442 (59\%) females and 998 (41\%) males, with a median age of 67 years (range 11-96). The histologic types included $1946(80 \%)$ adenocarcinomas; 324 (13\%) squamous cell carcinomas; 147 (6\%) poorly-differentiated non-small cell lung carcinomas, not otherwise specified; $12(0.5 \%)$ pleomorphic/giant cell/ spindle cell carcinomas; and $11(0.5 \%)$ adenosquamous carcinomas. Targeted next-generation sequencing was performed on 1531 (63\%) primary tumors, 848 (35\%) metastases, 36 (2\%) local recurrences, and 25 from unspecified sites. The cohort harbored mutations in KRAS and EGFR overall in $33 \%$ and $19 \%$ of cases, respectively, comparable with published data [39]. 


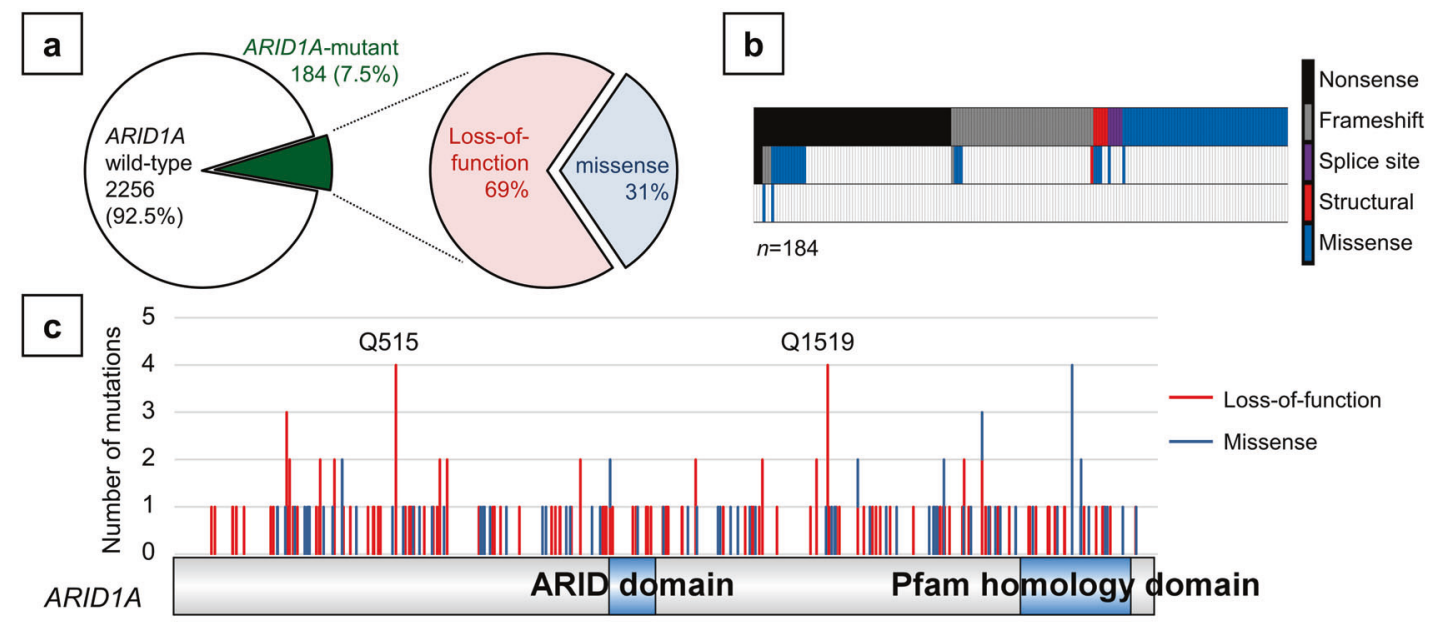

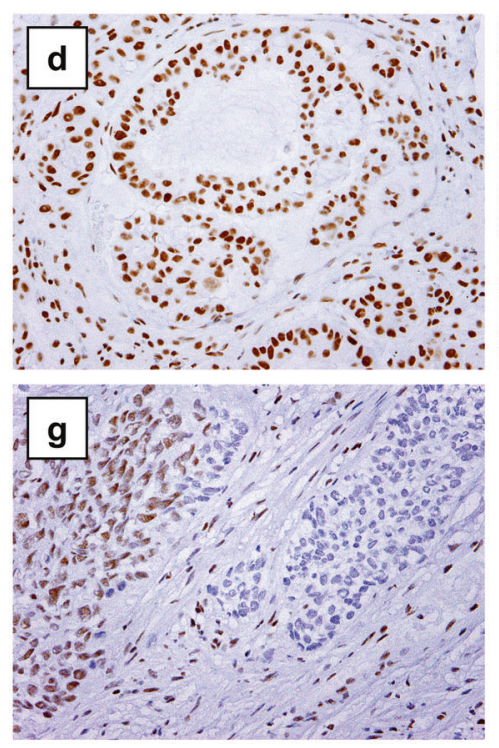

Fig. 1 Mutation spectrum and protein expression patterns of ARID1A in non-small cell lung carcinomas. a Pie chart showing the presence and spectrum of ARIDIA mutations in non-small cell lung carcinomas. b Heatmap showing the distribution of ARID1A alterations in non-small cell lung carcinomas (214 from 184 patients), with the types of alterations designated by their respective colors. c Location, number, and type (loss-of-function, red; missense, blue) of

\section{Mutations in ARID1A in non-small cell lung carcinomas}

ARIDIA mutations were detected in $184(7.5 \%)$ non-small cell lung carcinomas, of which $138(75 \%)$ were adenocarcinomas, $33(18 \%)$ were squamous cell carcinomas, and $13(7 \%)$ were other subtypes. LOF alterations were present in $127(69 \%)$ tumors (Fig. 1a), including nonsense mutations in $68(37 \%)$, frameshift mutations in $52(28 \%)$, splice site mutations in $6(3 \%)$, and structural rearrangement/ truncating mutations in $5(3 \%)$. Missense mutations were noted in 77 (42\%). Multiple ARIDIA mutations were present in 28 (15\%) tumors (Fig. 1b), including two mutations each in 26 tumors and three mutations in 2 tumors. The
ARID1A mutations, with hotspot loss-of-function mutations at Q515 and Q1519 $\left(P=2.3 \times 10^{-6}\right)$. ARID1A expression patterns included (d) intact, e diminished, $\mathbf{f}$ complete loss of expression in all tumor cells, $\mathbf{g}$ heterogeneous expression with loss in a subset of tumor cells in a geographic pattern, or (h-i) an interspersed pattern, with ARID1Adeficient cells interspersed among ARID1A-intact cells.

mutations were scattered throughout the ARIDIA gene, as expected for a tumor suppressor gene (Fig. 1c). Of all ARIDIA mutations in this study, $4(2 \%)$ each were located at Q515 (4 nonsense) and Q1519 (2 nonsense; 2 frameshift), representing recurrently affected positions in this gene $(P=$ $2.3 \times 10^{-6}<$ Bonferroni-corrected threshold $0.05 / 2286=$ $\left.2.2 \times 10^{-5}\right)$.

\section{Immunohistochemistry for ARID1A in non-small cell lung carcinomas}

Intact nuclear immunoreactivity (Fig. 1d), at an intensity commensurate with benign internal control cells, was observed in all 40 ARIDIA-wild-type lung tumors. Of 144 


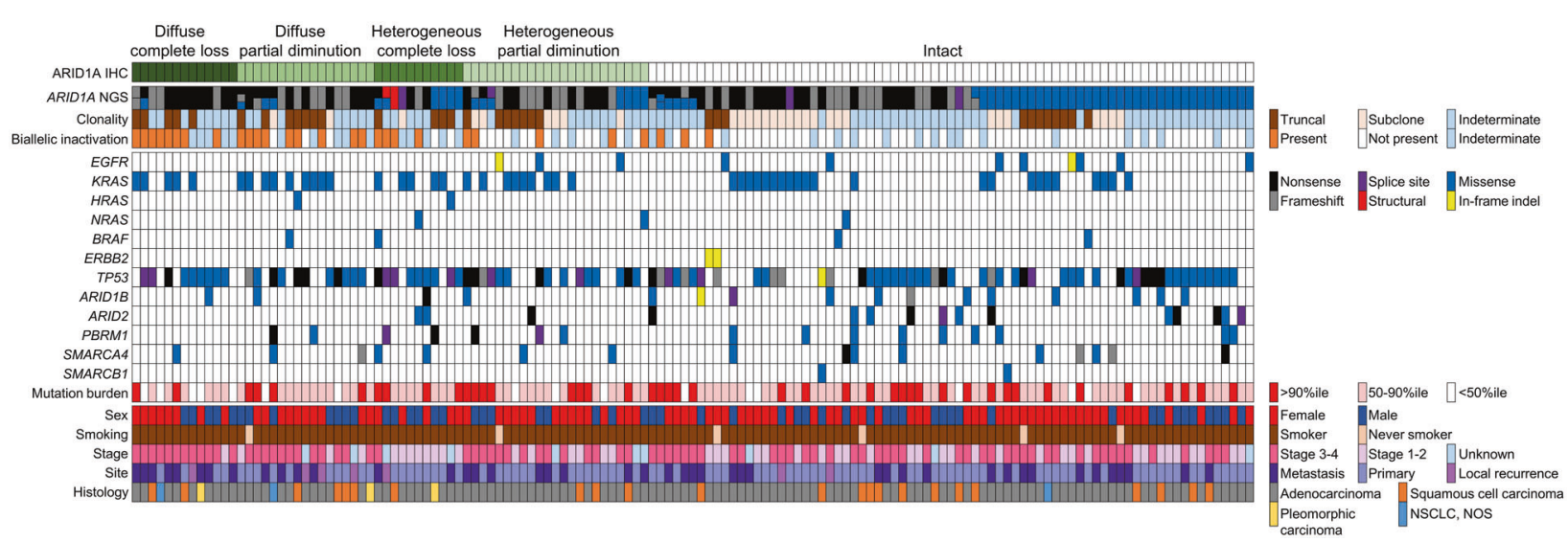

Fig. 2 Landscape of ARID1A protein expression and genomic alterations in 139 non-small cell lung carcinomas. The data are grouped by ARID1A expression patterns and include types and numbers of ARID1A mutations, ARIDIA mutation clonality assessment, and evidence of biallelic inactivation. Concurrent mutations in
EGFR, KRAS, HRAS, NRAS, BRAF, ERBB2, TP53, ARID1B, ARID2, PBRM1, SMARCA4, and SMARCB1 are displayed if present, along with tumor mutation burden, patients' sex, smoking history, clinical stage, tumor site sequenced, and histology.
(78\%) ARIDIA-mutant tumors tested, 5 had insufficient tumor cells or internal positive control (stromal or inflammatory cells) for scoring and were excluded from further analysis. Among the 139 quantifiable cases, ARID1A expression was intact in $75(54 \%)$ and aberrant in $64(46 \%)$ tumors. Patterns of aberrant expression included: diffuse diminished expression (Fig. 1e) in 17 (12\%) ARIDIAmutant tumors, diffuse complete loss (Fig. 1f) in $13(9 \%)$ tumors, and intratumoral heterogeneous loss of ARID1A expression in $34(25 \%)$ tumors. Heterogeneous patterns of loss of expression could be geographic, involving a contiguous area (Fig. 1g); or interspersed, with ARID1Adeficient cells interspersed among ARID1A-intact cells (Fig. 1h, i).

\section{Correlation between ARID1A mutation and expression status in non-small cell lung carcinomas}

The landscape of the 139 ARIDIA-mutant non-small cell lung carcinomas with corresponding ARID1A expression patterns, molecular profile, and clinicopathologic features is illustrated in Fig. 2. The list of ARIDIA mutations along with their corresponding clonality index, TMB, and ARID1A immunohistochemical patterns are provided in Supplementary Table 1. The relationship between ARIDIA mutation status and protein expression is complex. This is exemplified by three lung adenocarcinomas harboring an identical ARIDIA nonsense mutation Q515* (Supplementary Fig. 2a-c) but showing different ARID1A expression patterns, with diffuse complete loss, heterogeneous loss, and intact expression in one case each (Supplementary Fig. 2d-f).

To dissect the relationship between ARIDIA mutation and expression status (Table 1), we examined the following parameters: (1) quantity, types, and positions of mutations in ARIDIA; (2) clonality index to determine if an ARIDIA mutation is truncal or sub-clonal; and (3) evidence of biallelic inactivation (see "Methods"). We found complete loss or diminished ARID1A expression to be significantly associated with ARIDIA LOF mutations ( $88 \%$ vs. $55 \% ; P<$ 0.0001 ; Table 1) and evidence of biallelic inactivation $(59 \%$ vs. 5\%; $P<0.0001$; Table 1$)$. On the other hand, the presence of multiple ARIDIA mutations alone had no significant effect on the presence of aberrant expression $(20 \%$ vs. $9 \% ; P=0.09$; Table 1 ), nor could the positions of ARID1A LOF mutations within the gene explain the expression patterns (Supplementary Fig. 3). Notably, for the 13 non-small cell lung carcinomas with complete loss of ARID1A expression, each harbored one or multiple ARIDIA LOF mutations, all of which were truncal rather than sub-clonal, with evidence of biallelic inactivation identified in all cases for which these parameters could be assessed.

\section{ARID1A mutations and loss of expression correlate with higher tumor mutational burden}

We examined the clinical and functional significance of ARIDIA mutations in non-small cell lung carcinomas; their correlations are summarized in Table 2.

Compared with ARIDIA-wild-type tumors, ARIDIAmutant tumors showed similar distributions in age, gender, and histologic types. ARIDIA-mutant tumors were less likely to harbor $E G F R$ mutations ( $9 \%$ vs. $20 \% ; P=0.0003$ ) and more likely to harbor TP53 mutations (69\% vs. 52\%; $P<0.0001)$ and showed higher mutational burden than ARIDIA-wild-type tumors $(P<0.0001)$. Overall, $84 \%$ of ARIDIA-mutant tumors harbored greater than the median 
Table 1 Correlation between ARID1A protein expression and ARIDIA molecular alterations in ARID1A-mutant non-small cell lung carcinomas.
Table 2 Clinicopathologic and molecular characteristics of nonsmall cell lung carcinomas with ARIDIA mutations.

\begin{tabular}{|c|c|c|c|c|c|c|}
\hline $\begin{array}{l}\text { ARID1A } \\
\text { expression pattern }\end{array}$ & Intact & Aberrant & $\begin{array}{l}\text { Diffuse } \\
\text { complete loss }\end{array}$ & $\begin{array}{l}\text { Diffuse partial } \\
\text { diminution }\end{array}$ & $\begin{array}{l}\text { Heterogeneous } \\
\text { complete loss }\end{array}$ & $\begin{array}{l}\text { Heterogeneous } \\
\text { partial diminution }\end{array}$ \\
\hline Cases & 75 & 64 & 13 & 17 & 11 & 23 \\
\hline$\%(N=139)$ & $54 \%$ & $46 \%$ & $9 \%$ & $12 \%$ & $8 \%$ & $17 \%$ \\
\hline \multicolumn{7}{|l|}{ LOF mutation(s) } \\
\hline Present & 41 & 56 & 13 & 17 & 7 & 19 \\
\hline Evaluable cases & 75 & 64 & 13 & 17 & 11 & 23 \\
\hline$\%$ & $55 \%$ & $88 \%$ & $100 \%$ & $100 \%$ & $64 \%$ & $83 \%$ \\
\hline$P$-value (vs. intact) & - & $P<0.0001 *$ & $P=0.0013^{*}$ & $P=0.0002^{*}$ & $P=0.75$ & $P=0.03$ \\
\hline \multicolumn{7}{|l|}{ Multiple mutations } \\
\hline Present & 7 & 13 & 2 & 5 & 2 & 4 \\
\hline Evaluable cases & 75 & 64 & 13 & 17 & 11 & 23 \\
\hline$\%$ & $9 \%$ & $20 \%$ & $15 \%$ & $29 \%$ & $18 \%$ & $17 \%$ \\
\hline$P$ value (vs. intact) & - & $P=0.09$ & $P=0.62$ & $P=0.04$ & $P=0.32$ & $P=0.28$ \\
\hline \multicolumn{7}{|l|}{ Truncal mutation(s) } \\
\hline Present & 11 & 23 & 5 & 7 & 4 & 7 \\
\hline 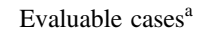 & 32 & 32 & 5 & 9 & 5 & 13 \\
\hline$\%$ & $34 \%$ & $72 \%$ & $100 \%$ & $78 \%$ & $80 \%$ & $54 \%$ \\
\hline$P$ value (vs. intact) & - & $P=0.005$ & $P=0.01$ & $P=0.03$ & $P=0.14$ & $P=0.32$ \\
\hline \multicolumn{7}{|l|}{ Biallelic inactivation } \\
\hline Present & 3 & 26 & 8 & 9 & 4 & 5 \\
\hline Evaluable cases ${ }^{\mathrm{b}}$ & 57 & 44 & 8 & 12 & 6 & 18 \\
\hline$\%$ & $5 \%$ & $59 \%$ & $100 \%$ & $75 \%$ & $67 \%$ & $28 \%$ \\
\hline$P$-value (vs. intact) & - & $P<0.0001 *$ & $P<0.0001 *$ & $P<0.0001^{*}$ & $P=0.0008^{*}$ & $P=0.02$ \\
\hline
\end{tabular}

*Significant $P$ value, as compared with tumors with intact expression after Bonferroni correction. ${ }^{\text {a }}$ Tumors harboring driver mutations in which ARIDIA mutation clonality index could be determined.

${ }^{\mathrm{b}}$ Tumors in which the status of biallelic inactivation could be determined.

\begin{tabular}{lcccc}
\hline ARIDIA & Wild-type & Mutant & LOF mutant & Non-LOF mutant \\
\hline Number & 2256 & 184 & 127 & 57 \\
Age: median (range) & $67(11-96)$ & $68(40-91)$ & $69(40-89)$ & $67(46-91)$ \\
Gender: no. (\%) & & & & \\
$\quad$ Female & $1331(59 \%)$ & $111(60 \%)$ & $76(60 \%)$ & $35(61 \%)$ \\
$\quad$ Male & $925(41 \%)$ & $73(40 \%)$ & $51(40 \%)$ & $22(39 \%)$ \\
Cases: no. (\%) & & & & \\
$\quad$ Primary & $1419(64 \%)$ & $112(61 \%)$ & $75(59 \%)$ & $37(66 \%)$ \\
Metastatic & $782(35 \%)$ & $66(36 \%)$ & $47(37 \%)$ & $19(34 \%)$ \\
Local recurrence & $31(1 \%)$ & $5(3 \%)$ & $5(4 \%)$ & $0(0 \%)$ \\
Histology: no. (\%) & $1808(80 \%)$ & $138(75 \%)$ & $94(74 \%)$ & $44(77 \%)$ \\
Adenocarcinoma & $291(13 \%)$ & $33(18 \%)$ & $24(19 \%)$ & $9(16 \%)$ \\
Squamous & $157(7 \%)$ & $13(7 \%)$ & $9(7 \%)$ & $4(7 \%)$ \\
Others & & & & \\
Mutation(s): no. (\%) & $449(20 \%)$ & $16(9 \%)^{\#}$ & $7(6 \%)^{\dagger}$ & $9(16 \%)$ \\
$E G F R$ & $1168(52 \%)$ & $127(69 \%)^{\dagger}$ & $83(65 \%)$ & $44(77 \%)^{\#}$ \\
TP53 & $741(33 \%)$ & $66(36 \%)$ & $50(39 \%)$ & $16(28 \%)$ \\
$\quad$ KRAS & $23.8(0.0-108.1)$ & $26.5(0.0-111.2)$ & $27.7(0.0-116.3)$ & $23.6(0.0-82.5)$ \\
Genomic instability index based on CNV: \\
median (10th-90th percentile)
\end{tabular}

$L O F$ loss-of-function, $T M B$ tumor mutational burden.

${ }^{\#} P \leq 0.0003$ and ${ }^{\dagger} P<0.0001$ as compared with ARIDIA wild-type (Bonferroni-corrected threshold $0.05 / 30=0.0016$ ).

number of mutations seen in our entire cohort of non-small cell lung carcinomas. Subgroup analysis demonstrated no significant differences in the mutational burden between tumors with ARIDIA LOF mutations and those with missense mutations $(P=0.54)$. ARIDlA mutations or aberrant ARID1A expression patterns were not associated with 
Table 3 Clinicopathologic and molecular characteristics of ARID1A-mutant non-small cell lung carcinomas with aberrant ARID1A expression.

\begin{tabular}{|c|c|c|c|c|}
\hline ARID1A expression & Intact & Loss & Diffuse loss & Heterogenous loss \\
\hline Number & 75 & 64 & 30 & 34 \\
\hline Age: median (range) & $68(42-91)$ & $68(40-91)$ & $69(40-88)$ & $68(43-91)$ \\
\hline \multicolumn{5}{|l|}{ Gender: no. (\%) } \\
\hline Female & $47(63 \%)$ & $38(59 \%)$ & $18(60 \%)$ & $20(59 \%)$ \\
\hline Male & $28(37 \%)$ & $26(41 \%)$ & $12(40 \%)$ & $14(41 \%)$ \\
\hline \multicolumn{5}{|l|}{ Smoking history } \\
\hline Smoker & $71(95 \%)$ & $62(97 \%)$ & $29(97 \%)$ & $33(97 \%)$ \\
\hline Never smoker & $4(5 \%)$ & $2(3 \%)$ & $1(3 \%)$ & $1(3 \%)$ \\
\hline \multicolumn{5}{|l|}{ Cases: no. (\%) } \\
\hline Primary & $51(68 \%)$ & $37(58 \%)$ & $14(47 \%)$ & $23(68 \%)$ \\
\hline Metastatic & $22(29 \%)$ & $22(34 \%)$ & $12(40 \%)$ & $10(29 \%)$ \\
\hline Local recurrence & $2(3 \%)$ & $5(8 \%)$ & $4(13 \%)$ & $1(3 \%)$ \\
\hline \multicolumn{5}{|l|}{ Overall survival } \\
\hline Median (years) & Not reached & 2.6 & 2.5 & 6.0 \\
\hline$P$ value & - & $P=0.03^{*}$ & $P=0.04 *$ & $P=0.10$ \\
\hline \multicolumn{5}{|l|}{ Histology: no. (\%) } \\
\hline Adenocarcinoma & $61(81 \%)$ & $49(77 \%)$ & $20(67 \%)$ & $29(85 \%)$ \\
\hline Squamous & $12(16 \%)$ & $10(16 \%)$ & $6(20 \%)$ & $4(12 \%)$ \\
\hline Others & $2(3 \%)$ & $5(8 \%)$ & $4(13 \%)$ & $1(3 \%)$ \\
\hline \multicolumn{5}{|l|}{ Mutation(s): no. (\%) } \\
\hline$E G F R$ & $8(11 \%)$ & $3(5 \%)$ & $0(0 \%)$ & $3(9 \%)$ \\
\hline TP53 & $52(69 \%)$ & $44(69 \%)$ & $20(67 \%)$ & $24(71 \%)$ \\
\hline$K R A S$ & $23(31 \%)$ & $29(45 \%)$ & $14(47 \%)$ & $14(41 \%)$ \\
\hline $\begin{array}{l}\text { Genomic instability index based on CNV: } \\
\text { median (10th-90th percentile) }\end{array}$ & $\begin{array}{l}24.3 \\
(0.0-107.8)\end{array}$ & $\begin{array}{l}22.1 \\
(0.0-113.4)\end{array}$ & $17.7(0.0-96.8)$ & $26.5(0.0-135.6)$ \\
\hline $\begin{array}{l}\text { TMB (per Mb): median (10th-90th } \\
\text { percentile) }\end{array}$ & $\begin{array}{l}19.3 \\
(10.8-38.0)\end{array}$ & $17.3(9.9-31.0)$ & $14.5(9.6-29.5)$ & $18.7(11.0-31.0)$ \\
\hline
\end{tabular}

TMB tumor mutational burden.

*Statistically significant as compared with tumors with intact ARID1A expression. All other comparisons statistically non-significant. mutations in other SWI/SNF members ARID1B, ARID2, SMARCA4, and SMARCB1, though PBRM1 mutations were more frequent in ARIDIA-mutant tumors (Supplementary Table 2).

For the 139 ARIDIA-mutant non-small cell lung carcinomas, the correlations of ARID1A expression status with clinicopathologic features are summarized in Table 3. Compared with the 75 ARID1A-intact tumors, the 64 tumors with aberrant expression showed similar distributions in age, gender, histologic types, mutations in EGFR/ TP53/KRAS, and mutational burdens; their mutational burden was significantly higher than that of ARIDIA-wild-type tumors $(P<0.0001)$.

Of note, in this cohort of 2440 non-small cell lung carcinoma patients, as expected, the presence of TP53 mutation was associated with increased copy number alterations and genomic instability (median index 31.2 vs. $18.2 ; P<0.0001$ ). However, there were no statistically significant differences in the genomic instability index between ARIDIA-wild-type and ARIDIA-mutant tumors (Table 2) and between ARID1Aintact tumors and tumors with aberrant ARID1A expression (Table 3).

\section{Loss of ARID1A protein expression, but not mutation, correlates with worse overall survival among ARID1A-mutant tumors}

In 2107 non-small cell lung carcinoma patients with available survival data, no significant differences in overall survival were noted for tumors harboring mutations in ARIDIA, ARIDIB, ARID2, or PBRM1 (Fig. 3a); however, survival was worse for patients with SMARCA4-mutant tumors, consistent with the published literature [18, 19, 21, 22]. Among the 125 patients with ARIDIA-mutant non-small cell lung carcinomas and available survival data, survival was similar among those with ARIDIA LOF versus missense variants (Fig. 3b), regardless of clinical stages (Supplementary Fig. 4a, b). In this group of patients, aberrant ARID1A expression was significantly associated with decreased overall survival (median 2.6 years vs. not reached; $P=0.03$; Fig. $3 \mathrm{c}$ and Table 3 ). Subgroup analysis revealed that survival differences persisted in advanced clinical stages III/IV (median 2.0 years vs. not reached; $P=0.014$; Supplementary Fig. $4 \mathrm{c}$ ) but not in stages I/II (median 6.0 years vs. not reached; $P=0.53$; Supplementary Fig. 4d); survival differences also persisted for 

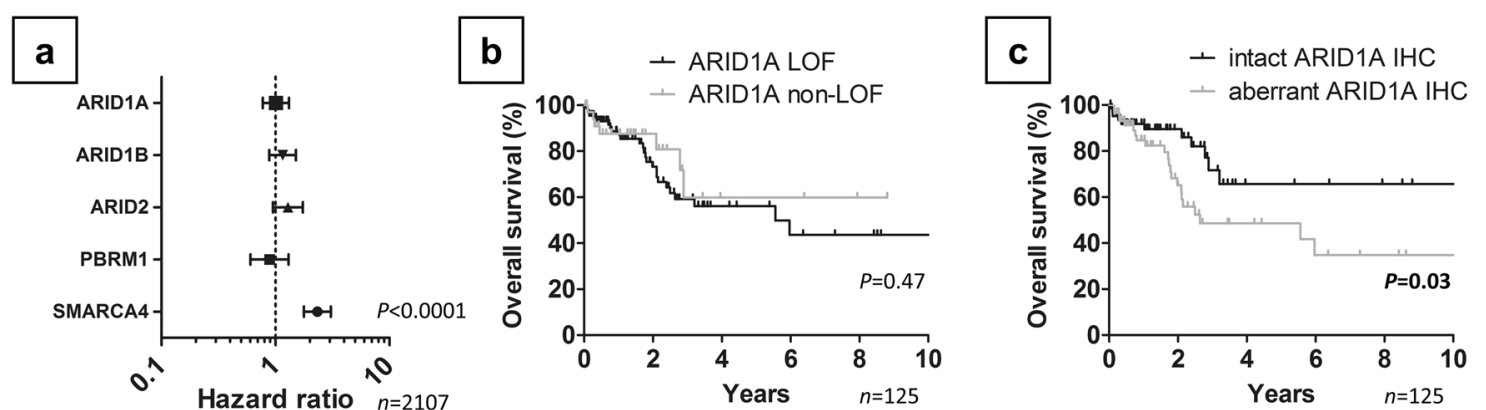

Fig. 3 Overall survival of non-small cell lung carcinoma patients with ARID1A mutations. a Forest plots of overall survival in 2107 patients with non-small cell lung carcinomas harboring mutations in ARID1A, ARID1B, ARID2, PBRM1, and SMARCA4, showing worse survival $(P<0.0001)$ only in SMARCA4-mutant tumors but not in

tumors with diffuse ARID1A loss (median 2.5 years vs. not reached; $P=0.04$; Table 3 ) but not for tumors with heterogeneous ARID1A loss (median 6.0 years vs. not reached; $P=0.10$; Table 3 ). Of note, the median overall survival was 2.1 years, 2.5 years, and 2.1 years for patients with tumors showing diffuse complete loss, diffuse diminished expression, and heterogeneous diminished expression of ARID1A, respectively; whereas the median overall survival was not reached for patients with tumors with intact expression or heterogeneous complete loss of ARID1A (Supplementary Fig. 4e).

\section{Diffuse loss of ARID1A expression in non-small cell lung carcinomas associated with poorly differentiated histology and frequent lack of driver mutations}

We next focused on the 30 non-small cell lung carcinomas with diffuse loss of ARID1A expression (Fig. 2 and Table 3). This group included 18 women and 12 men, with a median age of 69 (range 40-88); all but one (97\%) were smokers. Histologically, this group included 20 adenocarcinomas (including solid-predominant in 13 and acinar-predominant with minor solid or cribriform patterns in seven tumors; Fig. 4a-f), six squamous cell carcinomas (including one with focal pleomorphic features and complete loss of ARID1A expression in both squamous and pleomorphic components; Fig. 4g-k), two pleomorphic carcinomas, and two poorly differentiated non-small cell carcinomas, not otherwise specified. Overall, 27 (90\%) tumors had high-grade features. TTF-1 immunoreactivity was noted in 8 of 20 (40\%) tumors tested, including 7 of $13(54 \%)$ adenocarcinomas and one pleomorphic carcinoma.

Genetically, $14(47 \%)$ tumors harbored KRAS activating mutations (G12V in 4; G12C in 3; G12F, G12A, G12L, G12D, G13C, V14I, and Q61H in one tumor each), and an HRAS activating mutation G13V was present in one additional tumor. No driver mutations or rearrangements were other groups. Kaplan-Meier overall survival of 125 ARIDIA-mutant non-small cell lung carcinoma patients (b) harboring ARID1A loss-offunction (LOF) mutation as compared with non-LOF mutation; and c showing intact expression as compared with loss of ARID1A expression.

detected in the remaining $15(50 \%)$ tumors, including in 8 of $13(62 \%)$ tumors with complete ARID1A loss. Other genomic alterations included pathogenic TP53 mutations in $20(67 \%)$ tumors, including in $12(80 \%)$ tumors with no mitogenic driver mutations. A PBRM1 nonsense mutation and a SMARCA4 frameshift mutation were present in one case each (Supplementary Table 3). Compared with ARIDIA-wild-type tumors, non-small cell lung carcinomas with diffuse loss of ARID1A expression showed increased mutational burden.

\section{Diffuse loss of ARID1A expression in rare non-small cell lung carcinomas with MMR deficiency}

MMR pathway-deficient non-small cell lung carcinomas were rare, with only three $(0.12 \%)$ tumors identified in our cohort of 2440 patients. All three contained ARIDIA mutations. Indeed, MMR deficiency was significantly enriched in tumors with diffuse ARID1A loss (2 of 30; $6.7 \% ; \quad P<0.0001)$, involving a tumor with complete ARID1A loss and a tumor with diminished ARID1A expression. Both tumors demonstrated an MMR-deficient mutational signature with frequent homopolymer insertiondeletion mutations $(12.1-13.7 / \mathrm{Mb})$ and increased mutational burden $(49.4 / \mathrm{Mb}$; $>99$ th percentile). One MMRdeficient lung adenocarcinoma with an ARIDIA frameshift mutation showed intact ARID1A protein expression, suggesting this represented a hypermutation-related passenger event in this tumor.

\section{Heterogeneous loss of ARID1A expression in non- small cell lung carcinomas}

The 34 patients with heterogeneous ARID1A loss (Fig. 2 and Table 3) included 20 women and 14 men, with a median age of 68 (range 43-91); all except one (97\%) were smokers, and $85 \%$ of tumors were adenocarcinomas. This subgroup showed no significant differences in its clinicopathologic and 

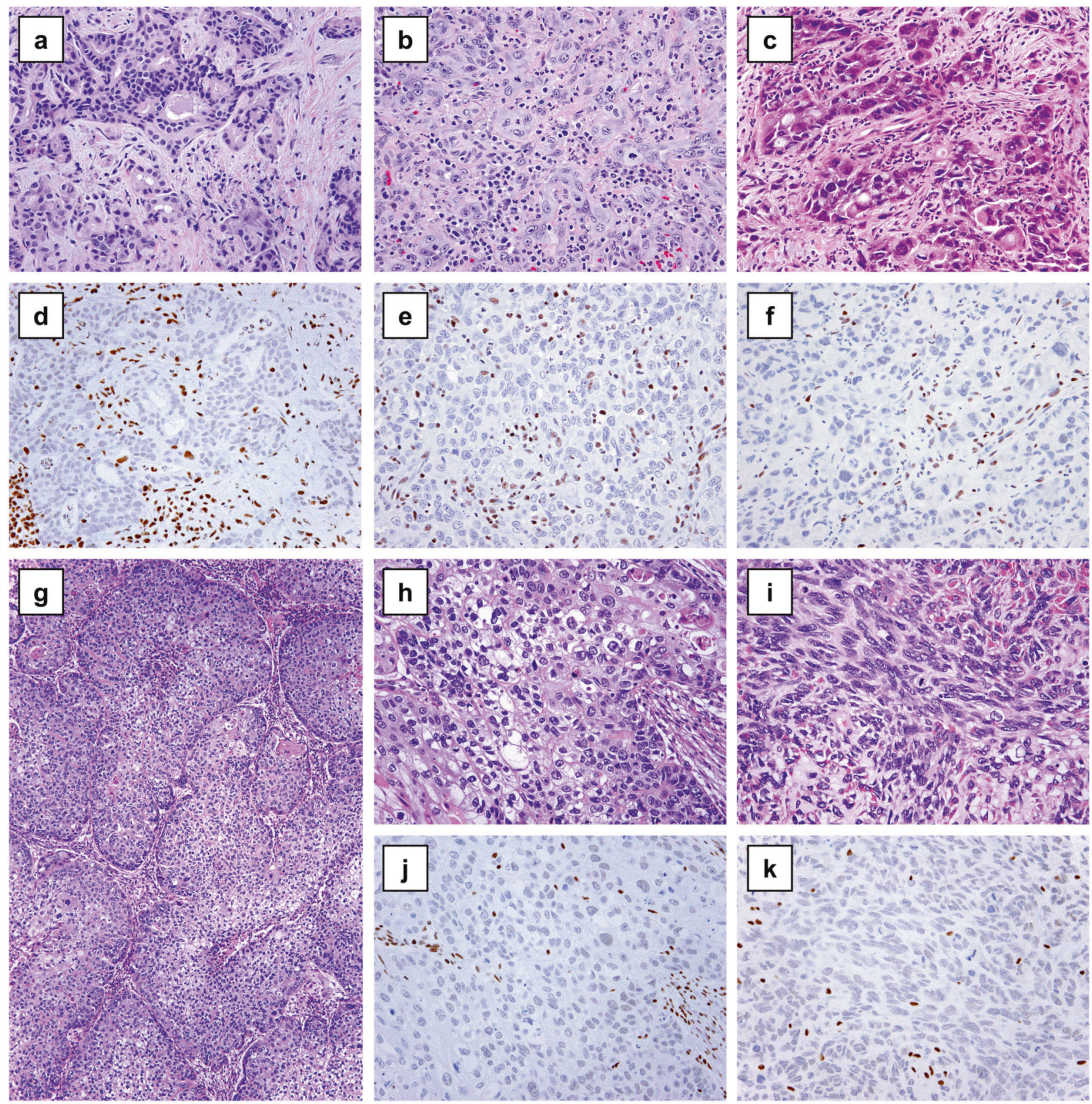

Fig. 4 Photomicrographs of ARID1A-deficient non-small cell lung carcinomas. a Metastatic lung adenocarcinoma (ARID1A Q474*; no known driver mutation) characterized by tumor cells with moderate eosinophilic cytoplasm and a cribriform pattern. b Lung adenocarcinoma (ARID1A S617*, KRAS G12F) in a solid architecture, with moderate amphophilic-to-vacuolated cytoplasm and brisk background chronic inflammation. c Metastatic lung adenocarcinoma (ARIDIA E1718* with loss of heterozygosity, KRAS G13C) showing a solid

molecular features as compared with ARIDIA-mutant tumors with intact or diffuse loss of ARID1A expression (Table 3), including no survival differences as compared with tumors with intact ARID1A expression $(P=0.10)$ or to those with diffuse ARID1A loss $(P=0.64)$. The patterns of heterogeneous ARID1A loss included complete loss in 11 architecture, prominent admixed neutrophils, and scattered mucin droplets. d-f All three tumors demonstrated complete loss of ARID1A expression. $\mathbf{g}-\mathbf{i}$ Lung squamous cell carcinoma (ARID1A S261* with loss of heterozygosity; no known driver mutation) composed of h squamoid-to-clear cells with focal keratinization, $\mathbf{i}$ along with a minor pleomorphic component with conspicuous mitoses and prominent spindling. $\mathbf{j}$, k Complete ARID1A expression loss was noted in both components.

(geographic in 3, interspersed in 8) and diminished expression in 23 tumors (geographic in 11, interspersed in 12). Geographic complete loss of ARID1A expression was notable in a squamous cell carcinoma and two adenocarcinomas, including one that showed high-grade fetal-like differentiation corresponding to areas of ARID1A loss (Fig. 5a-c). 


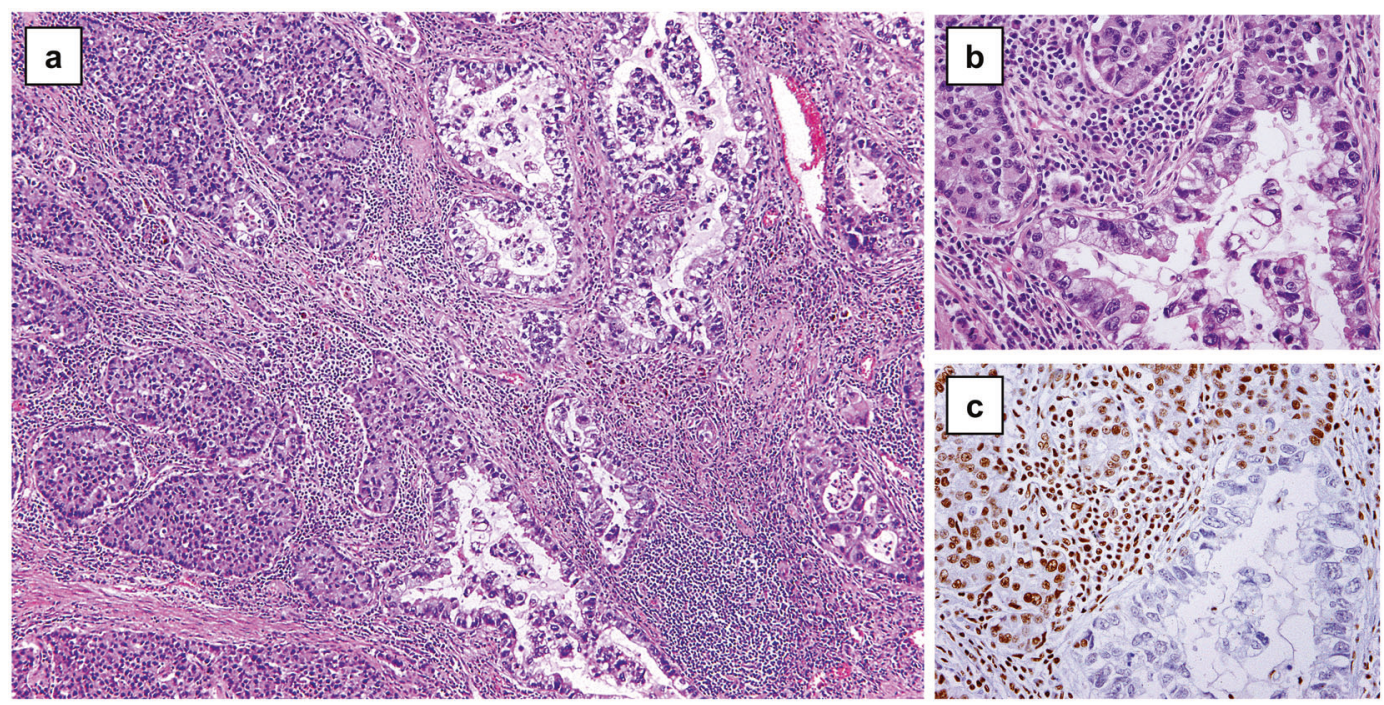

Fig. 5 Geographic loss of ARID1A protein expression in a lung adenocarcinoma showing focal high grade fetal-like differentiation. Photomicrographs of a lung adenocarcinoma (ARID1A Q2070*,
$K R A S \mathrm{G} 12 \mathrm{~V}$ ) at a low- and $\mathbf{b}$ high-power magnification, showing $\mathbf{c}$ heterogeneous geographic loss of ARID1A expression with corresponding high-grade fetal-like differentiation.

\section{Discussion}

In this large institutional cohort of 2440 non-small cell lung carcinomas, ARIDIA mutations were detected in $7.5 \%$; however, we estimated that only $1-2 \%$ of non-small cell lung carcinomas show corresponding loss of ARID1A protein expression, consistent with a recent report of ARID1A loss in 1.3\% of 1013 non-small cell lung carcinomas using tissue microarrays [17]. Aberrant ARID1A expression patterns included complete loss or diminished expression in a diffuse or heterogeneous pattern, the latter of which could be geographic or interspersed. While complete or heterogeneous geographic ARID1A loss has been described, the interspersed ARID1A loss pattern has not been previously reported to our knowledge. Aberrant ARID1A expression correlated with ARIDIA LOF mutations and evidence of biallelic inactivation. Both ARIDIA mutations and aberrant expression correlated with a lack of EGFR mutations, frequent TP53 mutations, and increased mutational burden. ARIDIA-mutant tumors showed similar overall survival compared with ARIDIA-wild-type tumors; however, among patients with ARIDIA-mutant tumors, ARID1A expression loss correlated with worse overall survival. Lung tumors with diffuse loss of ARID1A expression were predominantly adenocarcinomas, poorly differentiated (including pleomorphic carcinomas in two tumors), almost exclusively from smokers, lacking driver mutations in 50\% of cases, and enriched for MMR deficiency. Heterogeneous geographic ARID1A loss was notable in three tumors, including an adenocarcinoma showing fetal-like differentiation in areas with ARID1A loss.
This study was limited in its use of data from a single institution with potential selection/referral bias, tumor-only sequencing with germline variants confounding interpretation of somatic alterations, and the use of a targeted sequencing panel that limits confident assessment of variant clonality. ARIDIA also contains repetitive regions that are susceptible to sequencing artifacts and misalignment, rendering some variant interpretation challenging. Nonetheless, we reported ARIDIA alterations in non-small cell lung carcinomas with similar prevalence as other studies $[5,8,11,12,25,29]$.

Despite the association of aberrant ARID1A expression with ARIDIA LOF mutations and evidence of biallelic inactivation, we noted that ARIDIA mutation status alone was an unreliable predictor of ARID1A expression. This disconnect between ARIDIA mutation and expression status had been documented in a subset of endometrial adenocarcinomas [5] and MMR-stable EBV-infected gastric adenocarcinomas [9]. In endometrial adenocarcinomas, while ARIDIA mutations were early clonal events in only $25 \%$ of cases, loss of ARID1A expression was commonly seen in large subsets of both primary tumors and metastases [40]. The discordance between ARIDIA mutation status and its expression status in non-small cell lung carcinomas may be due to several technical and biological reasons: First, a substantial fraction $(47 \%)$ of ARIDIA mutations herein were sub-clonal, raising the possibility that at least some of these are passenger mutations of questionable functional relevance. Second, our data may be limited by tumoral heterogeneity, as a tumor with heterogeneous loss of expression may show only intact or complete-loss pattern in a limited sample; this bias was minimized by performing 
immunohistochemistry on whole-tissue sections rather than tissue microarrays. Third, loss of ARID1A expression may be due to posttranscriptional, posttranslational, and epigenetic mechanisms [1] not examined herein; however, in no case did we observe protein loss in the absence of a corresponding LOF mutation. Fourth, as we measured ARID1A expression as a correlate of $A R I D I A$ gene function, we did not capture deleterious effects from missense or in-frame indel variants that nonetheless have intact epitope. Rare missense mutations in SMARCA4 had been reported to disrupt chromatin remodeling by altering its conserved ATPase surfaces and the accessibility landscape of enhancers [41]. Nevertheless, our findings of ARIDIA mutational status being a poor predictor for ARID1A expression status may have implications for interpretation of ARIDIA sequence variants if ARID1A status is used in consideration of choice of therapy.

In describing the clinicopathologic and molecular features of non-small cell lung carcinomas with ARIDIA mutations or aberrant ARID1A expression, we noted the following observations:

First, our findings of frequent TP53 mutations in ARIDIAmutant non-small cell lung carcinomas were consistent with a recent analysis on plasma DNA from patients with advanced lung adenocarcinoma, in which $70 \%$ of patients with ARID1A-mutant tumors harbored concurrent TP53 mutations. Interestingly, this observation demonstrated a different genetic context and relationship between TP53 and ARIDIA alterations as compared with other tumors, as ARIDIA-mutant gastric, endometrial, and ovarian carcinomas typically harbored wild-type TP53 [9, 10, 42], while no relationship was apparent between ARID1A and p53 expression in esophageal adenocarcinoma [43].

Second, heterogeneous geographic ARID1A loss was notable in one adenocarcinoma showing high-grade fetallike differentiation. Though rare, lung adenocarcinomas with a high-grade fetal adenocarcinoma-like component harbored a poor prognosis [44]. The loss of ARID1A expression precisely in the fetal-like areas suggested that ARID1A may be a molecular correlate of this histologic transformation in some cases.

Third, while the association between ARID1A loss and microsatellite instability we observed in rare non-small cell lung carcinomas had been described in endometrial [42, 45, 46], ovarian [46], colorectal [47-49], and gastric adenocarcinomas [9, 10, 47], the mechanistic links between ARID1A loss and MMR deficiency varied by tumor types. ARID1A loss may represent a consequence of MMR deficiency, as ARIDIA contains repetitive sequences that are prone to mutagenesis, as seen in MMR-deficient gastric adenocarcinomas [9]. ARID1A loss is associated with MLH1 promoter hypermethylation resulting in sporadic MMR deficiency in a subset of colorectal [48] and endometrial adenocarcinomas [42]. ARID1A is also a binding partner of MSH2; loss of ARID1A can thus directly compromise MMR [31].

ARID1A loss has been associated with improved response to immunotherapy across diverse tumor types [31, 33, 50]. ARID1A can directly interact with EZH2 to antagonize EZH2-mediated interferon response [51]. Specific to nonsmall cell lung carcinomas, ARIDIA mutation status may predict improved response to durvalumab plus tremelimumab [32]. However, it is unclear whether ARIDIA mutation status or protein expression status is a better biomarker in predicting response in these patients. Further correlative studies of ARID1A may clarify its role in selecting patients who benefit from immune checkpoint blockade.

In summary, while ARIDIA mutations were present in $7.5 \%$ of non-small cell lung carcinomas, $<2 \%$ showed diffuse loss of ARID1A expression, which was associated with high-grade histologic features, frequent TP53 mutations, and lack of targetable driver mutations. With ARID1A emerging as a potential therapeutic target, our findings have implications for $A R I D 1 A$ variant interpretation in clinical sequencing assays. Functional characterization of alterations including in ARIDIA that contribute to the phenotypic spectrum of non-small cell lung carcinomas may enable better patient selection for personalized treatment.

Acknowledgements We thank the Center for Advanced Molecular Diagnostics and the Immunohistochemistry Laboratory in the Department of Pathology at the Brigham and Women's Hospital for technical support.

\section{Compliance with ethical standards}

Conflict of interest The authors report the following disclosures: LMS: Consulting/honorarium from EMD Serono, LOXO Oncology, AstraZeneca, and Foghorn Therapeutics and institutional research funding from Roche/Genentech; AR: Consulting/honorarium from AstraZeneca, Ariad Pharmaceuticals, Roche, Boehringer/Ingelheim, and Medtronic and institutional research funding from AstraZeneca.

Publisher's note Springer Nature remains neutral with regard to jurisdictional claims in published maps and institutional affiliations.

\section{References}

1. Wu JN, Roberts CW. ARID1A mutations in cancer: another epigenetic tumor suppressor. Cancer Discov. 2013;3:35-43.

2. Kadoch C, Hargreaves DC, Hodges C, Elias L, Ho L, Ranish J, et al. Proteomic and bioinformatic analysis of mammalian SWI/ SNF complexes identifies extensive roles in human malignancy. Nat Genet. 2013;45:592-601.

3. Jones S, Wang TL, Shih Ie M, Mao TL, Nakayama K, Roden R, et al. Frequent mutations of chromatin remodeling gene ARID1A in ovarian clear cell carcinoma. Science. 2010;330:228-31.

4. Wiegand KC, Shah SP, Al-Agha OM, Zhao Y, Tse K, Zeng T, et al. ARID1A mutations in endometriosis-associated ovarian carcinomas. N Engl J Med. 2010;363:1532-43.

5. Guan B, Mao TL, Panuganti PK, Kuhn E, Kurman RJ, Maeda D, et al. Mutation and loss of expression of ARID1A in uterine 
low-grade endometrioid carcinoma. Am J Surg Pathol. 2011;35: 625-32.

6. Fujimoto A, Totoki Y, Abe T, Boroevich KA, Hosoda F, Nguyen $\mathrm{HH}$, et al. Whole-genome sequencing of liver cancers identifies etiological influences on mutation patterns and recurrent mutations in chromatin regulators. Nat Genet. 2012;44:760-4.

7. Guichard C, Amaddeo G, Imbeaud S, Ladeiro Y, Pelletier L, Maad IB, et al. Integrated analysis of somatic mutations and focal copy-number changes identifies key genes and pathways in hepatocellular carcinoma. Nat Genet. 2012;44:694-8.

8. Lawrence MS, Stojanov P, Mermel CH, Robinson JT, Garraway LA, Golub TR, et al. Discovery and saturation analysis of cancer genes across 21 tumour types. Nature. 2014;505:495-501.

9. Wang K, Kan J, Yuen ST, Shi ST, Chu KM, Law S, et al. Exome sequencing identifies frequent mutation of ARID1A in molecular subtypes of gastric cancer. Nat Genet. 2011;43:1219-23.

10. Zang ZJ, Cutcutache I, Poon SL, Zhang SL, McPherson JR, Tao J, et al. Exome sequencing of gastric adenocarcinoma identifies recurrent somatic mutations in cell adhesion and chromatin remodeling genes. Nat Genet. 2012;44:570-4.

11. Imielinski M, Berger AH, Hammerman PS, Hernandez B, Pugh TJ, Hodis E, et al. Mapping the hallmarks of lung adenocarcinoma with massively parallel sequencing. Cell. 2012;150:1107-20.

12. Cancer Genome Atlas Research N. Comprehensive molecular profiling of lung adenocarcinoma. Nature. 2014;511:543-50.

13. Huang HN, Lin MC, Huang WC, Chiang YC, Kuo KT. Loss of ARID1A expression and its relationship with PI3K-Akt pathway alterations and ZNF217 amplification in ovarian clear cell carcinoma. Mod Pathol. 2014;27:983-90.

14. Berns K, Sonnenblick A, Gennissen A, Brohee S, Hijmans EM, Evers B, et al. Loss of ARID1A activates ANXA1, which serves as a predictive biomarker for trastuzumab resistance. Clin Cancer Res. 2016;22:5238-48.

15. Mathur R, Alver BH, San Roman AK, Wilson BG, Wang X, Agoston AT, et al. ARID1A loss impairs enhancer-mediated gene regulation and drives colon cancer in mice. Nat Genet. 2017;49: 296-302.

16. Sun X, Wang SC, Wei Y, Luo X, Jia Y, Li L, et al. Arid1a has context-dependent oncogenic and tumor suppressor functions in liver cancer. Cancer Cell. 2017;32:574-89 e6.

17. Naito T, Udagawa H, Umemura S, Sakai T, Zenke Y, Kirita K, et al. Non-small cell lung cancer with loss of expression of the SWI/SNF complex is associated with aggressive clinicopathological features, PD-L1-positive status, and high tumor mutation burden. Lung Cancer. 2019;138:35-42.

18. Rekhtman N, Montecalvo J, Chang JC, Alex D, Ptashkin RN, Ai N, et al. SMARCA4-deficient thoracic sarcomatoid tumors represent primarily smoking-related undifferentiated carcinomas rather than primary thoracic sarcomas. J Thorac Oncol. 2020;15:231-47.

19. Dagogo-Jack I, Schrock AB, Kem M, Jessop N, Lee J, Ali SM, et al. Clinicopathologic characteristics of BRG1-deficient NSCLC. J Thorac Oncol. 2020;15:766-76.

20. Le Loarer F, Watson S, Pierron G, de Montpreville VT, Ballet S, Firmin N, et al. SMARCA4 inactivation defines a group of undifferentiated thoracic malignancies transcriptionally related to BAF-deficient sarcomas. Nat Genet. 2015;47:1200-5.

21. Yoshida A, Kobayashi E, Kubo T, Kodaira M, Motoi T, Motoi N, et al. Clinicopathological and molecular characterization of SMARCA4-deficient thoracic sarcomas with comparison to potentially related entities. Mod Pathol. 2017;30:797-809.

22. Sauter JL, Graham RP, Larsen BT, Jenkins SM, Roden AC, Boland JM. SMARCA4-deficient thoracic sarcoma: a distinctive clinicopathological entity with undifferentiated rhabdoid morphology and aggressive behavior. Mod Pathol. 2017;30:1422-32.

23. Reisman DN, Sciarrotta J, Wang W, Funkhouser WK, Weissman BE. Loss of BRG1/BRM in human lung cancer cell lines and primary lung cancers: correlation with poor prognosis. Cancer Res. 2003;63:560-6.

24. Matsubara D, Kishaba Y, Ishikawa S, Sakatani T, Oguni S, Tamura $\mathrm{T}$, et al. Lung cancer with loss of BRG1/BRM, shows epithelial mesenchymal transition phenotype and distinct histologic and genetic features. Cancer Sci. 2013;104:266-73.

25. Yoshimoto T, Matsubara D, Nakano T, Tamura T, Endo S, Sugiyama $\mathrm{Y}$, et al. Frequent loss of the expression of multiple subunits of the SWI/SNF complex in large cell carcinoma and pleomorphic carcinoma of the lung. Pathol Int. 2015;65:595-602.

26. Bell EH, Chakraborty AR, Mo X, Liu Z, Shilo K, Kirste S, et al. SMARCA4/BRG1 is a novel prognostic biomarker predictive of cisplatin-based chemotherapy outcomes in resected non-small cell lung cancer. Clin Cancer Res. 2016;22:2396-404.

27. Agaimy A, Fuchs F, Moskalev EA, Sirbu H, Hartmann A, Haller F. SMARCA4-deficient pulmonary adenocarcinoma: clinicopathological, immunohistochemical, and molecular characteristics of a novel aggressive neoplasm with a consistent TTF1(neg)/ CK7(pos)/HepPar-1(pos) immunophenotype. Virchows Arch. 2017;471:599-609.

28. Zutter MM, Bloom KJ, Cheng L, Hagemann IS, Kaufman JH, Krasinskas AM, et al. The Cancer Genomics Resource List 2014. Arch Pathol Lab Med. 2015;139:989-1008.

29. Karachaliou N, Paulina Bracht JW, Rosell R. ARID1A gene driver mutations in lung adenocarcinomas. J Thorac Oncol. 2018; 13:e255-7.

30. Bitler BG, Aird KM, Garipov A, Li H, Amatangelo M, Kossenkov $\mathrm{AV}$, et al. Synthetic lethality by targeting EZH2 methyltransferase activity in ARID1A-mutated cancers. Nat Med. 2015;21:231-8.

31. Shen J, Ju Z, Zhao W, Wang L, Peng Y, Ge Z, et al. ARID1A deficiency promotes mutability and potentiates therapeutic antitumor immunity unleashed by immune checkpoint blockade. Nat Med. 2018;24:556-62.

32. Rizvi N, Cho BC, Reinmuth N, Lee KH, Luft A, Ahn M, et al. Mutations associated with sensitivity or resistance to immunotherapy in MNSCLC: analysis from the MYSTIC trial. In: World Conference on Lung Cancer. Barcelona, Spain: International Association for the Study of Lung Cancer; 2019.

33. Okamura R, Kato S, Lee S, Jimenez RE, Sicklick JK, Kurzrock R. ARID1A alterations function as a biomarker for longer progression-free survival after anti-PD-1/PD-L1 immunotherapy. J Immunother Cancer. 2020;8:pii:e000438.

34. Sholl LM, Do K, Shivdasani P, Cerami E, Dubuc AM, Kuo FC, et al. Institutional implementation of clinical tumor profiling on an unselected cancer population. JCI Insight. 2016;1:e87062.

35. Garcia EP, Minkovsky A, Jia Y, Ducar MD, Shivdasani P, Gong $\mathrm{X}$, et al. Validation of OncoPanel: a targeted next-generation sequencing assay for the detection of somatic variants in cancer. Arch Pathol Lab Med. 2017;141:751-8.

36. Walter MJ, Shen D, Shao J, Ding L, White BS, Kandoth C, et al. Clonal diversity of recurrently mutated genes in myelodysplastic syndromes. Leukemia. 2013;27:1275-82.

37. Nowak JA, Yurgelun MB, Bruce JL, Rojas-Rudilla V, Hall DL, Shivdasani P, et al. Detection of mismatch repair deficiency and microsatellite instability in colorectal adenocarcinoma by targeted next-generation sequencing. J Mol Diagn. 2017;19:84-91.

38. Liu W, Xie Y, Ma J, Luo X, Nie P, Zuo Z, et al. IBS: an illustrator for the presentation and visualization of biological sequences. Bioinformatics. 2015;31:3359-61.

39. Sholl LM, Aisner DL, Varella-Garcia M, Berry LD, Dias-Santagata D, Wistuba II, et al. Multi-institutional oncogenic driver mutation analysis in lung adenocarcinoma: the lung cancer mutation consortium experience. J Thorac Oncol. 2015;10:768-77.

40. Gibson WJ, Hoivik EA, Halle MK, Taylor-Weiner A, Cherniack $\mathrm{AD}$, Berg A, et al. The genomic landscape and evolution of 
endometrial carcinoma progression and abdominopelvic metastasis. Nat Genet. 2016;48:848-55.

41. Hodges HC, Stanton BZ, Cermakova K, Chang CY, Miller EL, Kirkland JG, et al. Dominant-negative SMARCA4 mutants alter the accessibility landscape of tissue-unrestricted enhancers. Nat Struct Mol Biol. 2018;25:61-72.

42. Bosse T, ter Haar NT, Seeber LM, v Diest PJ, Hes FJ, Vasen HF, et al. Loss of ARID1A expression and its relationship with PI3KAkt pathway alterations, TP53 and microsatellite instability in endometrial cancer. Mod Pathol. 2013;26:1525-35.

43. Drage MG, Tippayawong M, Agoston AT, Zheng Y, Bueno R, Hornick JL, et al. Morphological features and prognostic significance of ARID1A-deficient esophageal adenocarcinomas. Arch Pathol Lab Med. 2017;141:970-7.

44. Suzuki M, Nakatani Y, Ito H, Narimatsu H, Yamada K, Yoshioka E, et al. Pulmonary adenocarcinoma with high-grade fetal adenocarcinoma component has a poor prognosis, comparable to that of micropapillary adenocarcinoma. Mod Pathol. 2018;31:1404-17.

45. Allo G, Bernardini MQ, Wu RC, Shih Ie M, Kalloger S, Pollett A, et al. ARID1A loss correlates with mismatch repair deficiency and intact p53 expression in high-grade endometrial carcinomas. Mod Pathol. 2014;27:255-61.
46. Coatham M, Li X, Karnezis AN, Hoang LN, Tessier-Cloutier B, Meng B, et al. Concurrent ARID1A and ARID1B inactivation in endometrial and ovarian dedifferentiated carcinomas. Mod Pathol. 2016;29:1586-93.

47. Jones S, Li M, Parsons DW, Zhang X, Wesseling J, Kristel P, et al. Somatic mutations in the chromatin remodeling gene ARID1A occur in several tumor types. Hum Mutat. 2012;33:100-3.

48. Ye J, Zhou Y, Weiser MR, Gonen M, Zhang L, Samdani T, et al. Immunohistochemical detection of ARID1A in colorectal carcinoma: loss of staining is associated with sporadic microsatellite unstable tumors with medullary histology and high TNM stage. Hum Pathol. 2014;45:2430-6.

49. Chou A, Toon CW, Clarkson A, Sioson L, Houang M, Watson N, et al. Loss of ARID1A expression in colorectal carcinoma is strongly associated with mismatch repair deficiency. Hum Pathol. 2014;45:1697-703.

50. Le DT, Durham JN, Smith KN, Wang H, Bartlett BR, Aulakh LK, et al. Mismatch repair deficiency predicts response of solid tumors to PD-1 blockade. Science. 2017;357:409-13.

51. Li J, Wang W, Zhang Y, Cieslik M, Guo J, Tan M, et al. Epigenetic driver mutations in ARID1A shape cancer immune phenotype and immunotherapy. J Clin Investig. 2020;130:2712-26. 